\title{
Endovascular Treatment for Middle Cerebral Artery Aneurysms: Single-Center Experience and Review of Literatures
}

Noriyuki Kijima, ${ }^{1,2}$ Shimpei Miura, ${ }^{1}$ Eisaku Terada, ${ }^{1}$ Ryota Nakagawa, ${ }^{1}$ Tetsuro Tachi, ${ }^{1}$ Koki Murakami, ${ }^{1}$ Yoshiko Okita,${ }^{1}$ Yonehiro Kanemura, ${ }^{1,3}$ Shin Nakajima, ${ }^{1}$ and Toshiyuki Fujinaka ${ }^{1}$

Objective: The efficacy of endovascular treatment for middle cerebral artery (MCA) aneurysms remains controversial. However, recent studies have reported the safety of endovascular treatment for MCA aneurysms. In this study, we studied the efficacy and clinical outcomes of endovascular treatment for MCA aneurysms in our hospital and the morphology and anatomy of MCA aneurysms that were suitable for endovascular treatment.

Methods: We retrospectively analyzed 26 cases of MCA aneurysms which had undergone endovascular treatment at our institution between January 2015 and October 2018. We studied sizes and shapes of the aneurysms, clinical and angiographical outcomes one year after the treatment, and complications in these 26 patients. We also compared the differences in these parameters of the 26 patients with those of 61 other patients who were treated with clipping during the same period.

Results: The median aneurysm size was $6.1 \mathrm{~mm}(1.8-29.9 \mathrm{~mm})$, with the shapes of the aneurysms irregular in 8, and round in the other 18 cases. Four cases (15.4\%) had ruptured aneurysms. All aneurysms were treated with assist techniques; 8 (30.8\%) were treated by stent-assisted technique and $18(69.2 \%)$ were treated by balloon-assisted technique and endovascular treatment was successfully performed in all $(100 \%)$ cases. While the aneurysms were completely obliterated in 22 of them (84.6\%), the remaining 4 cases $(15.4 \%)$ had neck remnants. We observed periprocedural complications in 5 of the $26(19.2 \%)$ aneurysms, all of which were transient and completely recovered during the follow-up period. The efficacy and complication rates were not different from the MCA aneurysms treated with clipping. All MCA aneurysms arising from the M1 trunk were treated with endovascular treatment, and those with a round shape with the axis not deviating from M1 were also treated with endovascular treatment.

Conclusion: Endovascular treatment for MCA aneurysms is safe and effective together with adjunctive techniques such as balloon-assisted technique or stent-assisted technique. Thus, M1 trunk aneurysms and MCA bifurcation aneurysms with a round shape along the same axis of MCA may be good indications for endovascular treatment. However, long-term clinical and angiographical outcomes remain unknown. Thus, further studies are needed to address the existing limitations.

Keywords > middle cerebral artery aneurysm, coil embolization

$\overline{{ }^{1} \text { Department of Neurosurgery, Osaka National Hospital, National }}$ Hospital Organization, Osaka, Osaka, Japan

${ }^{2}$ Department of Neurosurgery, Osaka University Graduate School of Medicine, Suita, Osaka, Japan

${ }^{3}$ Department of Biomedical Research and Innovation, Institute for Clinical Research, National Hospital Organization Osaka National Hospital, Osaka, Osaka, Japan

Received: June 27, 2019; Accepted: August 12, 2020 Corresponding author: Noriyuki Kijima. Department of Neurosurgery, Osaka University Graduate School of Medicine, 2-2, Yamadaoka, Suita, Osaka 565-0871, Japan Email:n-kijima@nsurg.med.osaka-u.ac.jp

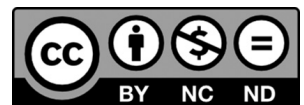

This work is licensed under a Creative Commons Attribution-NonCommercialNoDerivatives International License.

(C)2021 The Japanese Society for Neuroendovascular Therapy

\section{Introduction}

The efficacy of endovascular treatment for middle cerebral artery (MCA) aneurysms remains controversial and clipping remains the standard treatment strategy in many institutions. This is because MCA aneurysms usually have complex shapes, such as a wide neck and incorporate the proximal part of M2, thereby rendering them unsuitable for endovascular treatment. ${ }^{1,2)}$ Furthermore, a surgical approach for MCA aneurysms is straightforward and previous studies have reported excellent outcomes of clipping the MCA aneurysms. $^{3-6)}$

However, recent advancements in endovascular techniques such as balloon-assisted and stent-assisted techniques 


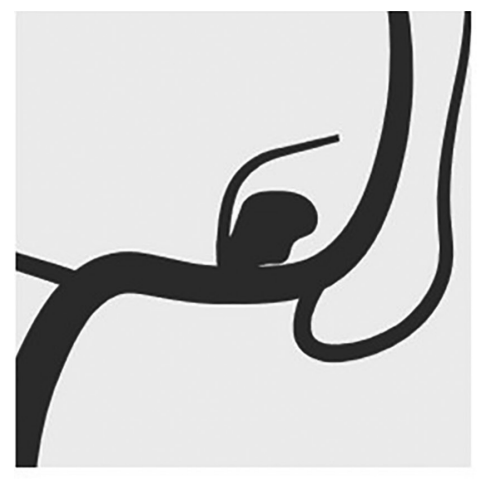

TypeI

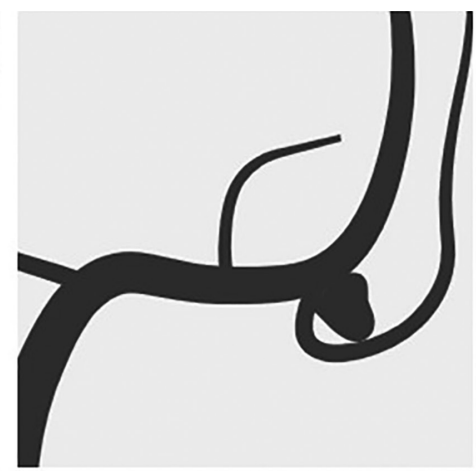

TypeII

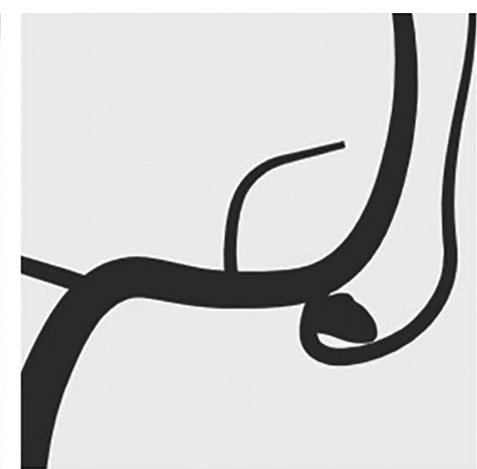

TypeIII

Fig. 1 Classification of MCA aneurysms; Type I: M1 trunk aneurysms, Type II: M1 bifurcation aneurysms located in the same axis of the M1 trunk, Type III: M1 bifurcation aneurysms with an axis deviating from the M1 trunk. MCA: middle cerebral artery

have made it possible to effectively perform endovascular treatment for aneurysms with complex shapes as described by recent reports. ${ }^{1,7,8)}$

In this study, we analyzed morphology and anatomy of MCA aneurysms that were suitable for endovascular treatment and compared the clinical and angiographical outcomes of endovascular treatment with surgical clipping.

\section{Materials and Methods}

\section{Patient sample size}

We retrospectively analyzed all cases of MCA aneurysms treated by endovascular procedure at our institution between January 2015 and October 2018. During this period, 87 cases of MCA aneurysms were treated, out of which 26 were treated by endovascular treatment.

\section{Indications for endovascular treatment and con- siderations}

The indications for endovascular treatment were as follows: MCA aneurysms located at M1 trunk, round-shaped and axis not deviating from M1 trunk, and patients without large intra-parenchymal hematoma in cases with a ruptured MCA aneurysm. To provide consideration and autonomy to patients, we also explained the details of both endovascular treatment and clipping and the treatment was selected based on patients' decision. We routinely use balloon-assisted technique for coiling intracranial aneurysms regardless of their locations in case of intra-procedural rupture.

\section{Case analysis and follow-up study}

We studied the morphology and anatomy of aneurysms, clinical and angiographical outcomes, and complication rate of endovascular treatment for MCA aneurysms. The differences in the aforementioned parameters were compared to those treated with surgical clipping. For analyzing the angiographical outcome, we performed an angiography one year after the treatment for endovascularly treated patients and a 3D computed tomography angiography (3D-CTA) a week and one year after surgery for clipping patients. Re-treatment or follow-up was based on the angiographical findings one year after the initial treatment. Angiographical outcomes were scored using Raymond Roy Occlusion Classification; complete obliteration, residual neck, and residual aneurysm. ${ }^{9)}$ To study the MCA aneurysms suitable for endovascular treatment, we classified anatomy of MCA aneurysm into three categories; Type I: M1 trunk aneurysms, Type II: M1 bifurcation aneurysms located in the same axis of the M1 trunk, Type III: M1 bifurcation aneurysms with axis deviating from the M1 trunk (Fig. 1).

\section{Results}

\section{Characteristics of MCA aneurysms treated by endovascular treatment}

The characteristics of 26 aneurysms are summarized in Table 1. The shape of the aneurysms was irregular in 8 $(30.8 \%)$ and round in $18(69.2 \%)$ cases. Four of the 26 (15.4\%) were ruptured aneurysms.

Endovascular treatment was successfully performed in all $(100 \%)$ cases and the aneurysms were completely obliterated in 22 of the 26 cases (84.6\%) while the remaining four cases (15.4\%) had neck remnants. The median follow-up period was 332 days (37-2191 days) and follow-up angiographic images were available in 15 of the 26 cases (57.7\%). 
Table 1 Characteristics for MCA aneurysms treated by coil embolization

\begin{tabular}{|c|c|c|}
\hline & Ruptured & Unruptured \\
\hline Age (average) & $50-59(55)$ & $47-80(68.0)$ \\
\hline \multicolumn{3}{|l|}{ Sex } \\
\hline Male & 3 & 5 \\
\hline Female & 1 & 17 \\
\hline \multicolumn{3}{|l|}{ Assist technique } \\
\hline Balloon & 4 & 13 \\
\hline Stent & 0 & 9 \\
\hline Complication & $0 \%$ & $22.7 \%(5 / 22)$ \\
\hline \multicolumn{3}{|l|}{ Initial occlusion status } \\
\hline Complete occlusion & 3 & 19 \\
\hline Neck remnant & 1 & 3 \\
\hline Residual aneurysm & 0 & 0 \\
\hline \multicolumn{3}{|c|}{ Occlusion status at follow-up } \\
\hline Complete occlusion & 3 & 12 \\
\hline Neck remnant & 0 & 0 \\
\hline Residual aneurysm & 0 & 0 \\
\hline $\mathrm{N} / \mathrm{A}$ & 1 & 10 \\
\hline \multicolumn{3}{|l|}{ mRS at last follow-up } \\
\hline 0 & 2 & 19 \\
\hline $1-2$ & 0 & 3 \\
\hline $3-5$ & 2 & 0 \\
\hline 6 & 0 & 0 \\
\hline
\end{tabular}

F: female; M: male; MCA: middle cerebral artery; mRS: modified Rankin Scale; N/A: not available

All the cases of aneurysms with follow-up angiographic images were confirmed to be completely obliterated.

We observed peri-procedural complications in 5 (19.2\%) aneurysms, with all the complications transient resolving completely. Two patients experienced intra-procedural rupture which did not result in serious complications. Another two patients experienced transient symptomatic ischemic complications with complete recovery.

One patient developed mild hemiparesis 3 months after endovascular treatment, with multiple contrast enhancing spot lesions in the distal territory of the treated aneurysm on brain MRI. The patient made full recovery by steroid therapy. All aneurysms were treated with assist techniques; 8 (30.8\%) were treated by stent-assisted technique and 18 (69.2\%) were treated by balloon-assisted technique. We observed no differences in success rate and complication rate between stent-assisted technique and balloon-assisted technique.

\section{Comparison with MCA aneurysms treated with clipping}

We compared the differences in patient's characteristics, aneurysm morphology, and aneurysm anatomy between
MCA aneurysms treated with endovascular treatment and those with clipping. We could not find any statistically significant differences in patient age, sex, size of the aneurysm, dome-neck ratio, and the existence of blebs between the endovascular and clipping groups (Table 2). The proportion of cases with M2 incorporation was lower in endovascular treatment group (31.8\%) compared with clipping group (44.3\%). The procedure success rate was $100 \%$ in all cases treated with either endovascular treatment or clipping. The rates of complete obliteration of the aneurysms were $84.6 \%(22 / 26)$ and $96.7 \%(59 / 61)$ in endovascular treatment and clipping groups, respectively. The re-treatment rate was $0 \%$ in either treatment modality. The complication rates were $19.2 \%(5 / 26)$ and $13.1 \%(8 / 61)$ in the endovascular treatment and clipping groups, respectively. The permanent morbidity rates were $0 \%(0 / 26)$ and $3.3 \%(2 / 61)$ in the endovascular treatment group and clipping group, respectively. The mortality rates of both procedures were 0\% (Table 3).

We further analyzed the differences in aneurysm anatomy relative to M1 and M2 between the two groups. We classified the morphology of MCA aneurysms into three categories as described above. We found that all five MCA aneurysms with Type I morphology were treated by endovascular treatment. Among MCA aneurysms with Type II morphology, 13/39 (33.3\%) MCA aneurysms with round shape and 4/20 (20\%) MCA aneurysms with irregular shape were treated with endovascular treatment. Among MCA aneurysms with Type III morphology, only 4/23 (17.4\%) MCA aneurysms were treated with endovascular treatment. (Table 4).

\section{Representative Cases}

\section{Case 1}

A 75-year-old woman with unruptured MCA aneurysm was treated with the stent-assisted technique. The aneurysm size was $2.9 \mathrm{~mm}$, but the neck of the aneurysm was $3.9 \mathrm{~mm}$. The patient was asymptomatic and refused to undergo the clipping procedure. Thus, we performed endovascular treatment and the procedure was performed by jail technique. We inserted an SL-10 at an angle of $45^{\circ}$ to the inferior trunk of M2 and we also inserted another SL-10 at an angle of $45^{\circ}$ into the aneurysm. Next, we deployed the Neuroform Atlas $3.0 \mathrm{~mm} \times 21 \mathrm{~mm}$ from the SL-10 which was inserted to M2. The stent was deployed from inferior trunk of M2 to the middle M1 to cover the neck of the aneurysm. We used Target 360 SOFT $3.0 \mathrm{~mm} \times 8 \mathrm{~cm}$ as 
Table 2 Comparison between MCA aneurysms treated by coil embolization and those treated by surgical clipping

\begin{tabular}{|c|c|c|c|c|c|c|}
\hline & \multicolumn{3}{|c|}{ Coil embolization } & \multicolumn{3}{|c|}{ Clipping } \\
\hline & Unruptured & Ruptured & Total & Unruptured & Ruptured & Total \\
\hline Number of cases & 22 & 4 & 26 & 49 & 12 & 61 \\
\hline $\mathrm{Age}^{*}$ & $68.0 \pm 12.0$ & $55.0 \pm 3.7$ & $66.0 \pm 12.1$ & $62.3 \pm 12.7$ & $63.3 \pm 15.0$ & $62.5 \pm 13.1$ \\
\hline Sex (proportion of females) & $77.3 \%$ & $25.0 \%$ & $69.2 \%$ & $67.3 \%$ & $66.7 \%$ & $67.2 \%$ \\
\hline Aneurysm size $(\mathrm{mm})^{*}$ & $4.57 \pm 1.93$ & $14.4 \pm 11.3$ & $6.08 \pm 5.61$ & $5.03 \pm 2.15$ & $6.48 \pm 2.88$ & $5.32 \pm 2.37$ \\
\hline Dome/neck ratio* & $1.33 \pm 0.29$ & $3.85 \pm 4.34$ & $1.71 \pm 1.79$ & $1.46 \pm 0.57$ & $1.50 \pm 0.43$ & $1.47 \pm 0.54$ \\
\hline Proportion of cases with bleb & $59.0 \%$ & $50.0 \%$ & $57.7 \%$ & $48.9 \%$ & $66.7 \%$ & $52.5 \%$ \\
\hline $\begin{array}{l}\text { Proportion of cases with M2 } \\
\text { incorporation }\end{array}$ & $27.8 \%$ & $50.0 \%$ & $31.8 \%$ & $42.9 \%$ & $50.0 \%$ & $44.3 \%$ \\
\hline
\end{tabular}

*Mean \pm 1 SD. MCA: middle cerebral artery.

Table 3 Difference in outcomes between MCA aneurysms treated by coil embolization and those treated by surgical clipping

\begin{tabular}{lcc} 
& Coil embolization & Clipping \\
Success rate & & \\
Complete occlusion & $84.6 \%(22 / 26)$ & $96.7 \%(59 / 61)$ \\
$\quad$ Neck remnant & $15.4 \%(4 / 26)$ & $3.3 \%(2 / 61)$ \\
Complication rate & $19.2 \%(5 / 26)$ & $13.1 \%(8 / 61)$ \\
Morbidity rate & $3.8 \%(1 / 26)$ & $3.3 \%(2 / 61)$ \\
Mortality rate & $0 \%(0 / 26)$ & $0 \%(0 / 61)$ \\
Retreatment rate & $0 \%(0 / 26)$ & $0 \%(0 / 61)$ \\
\hline
\end{tabular}

Table 4 Shape of MCA aneurysms treated by coil embolization or surgical clipping

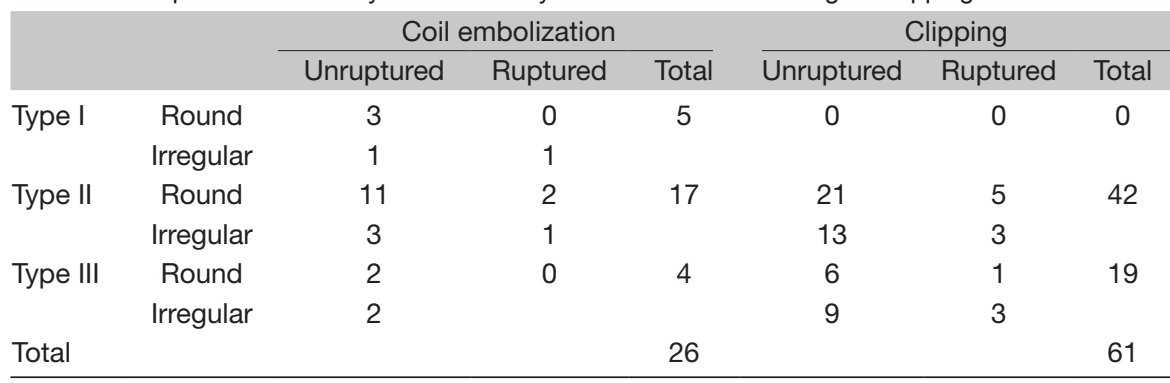

MCA: middle cerebral artery

first coil and then used Target 360 ULTRA $2.0 \mathrm{~mm} \times 3 \mathrm{~cm}$, Target 360 ULTRA $2.0 \mathrm{~mm} \times 3 \mathrm{~cm}$, Target 360 NANO 1.0 $\mathrm{mm} \times 3 \mathrm{~cm}$, Target 360 NANO $1.0 \mathrm{~mm} \times 3 \mathrm{~cm}$, and Target 360 NANO $1.0 \mathrm{~mm} \times 2 \mathrm{~cm}$. The aneurysm was completely obliterated at the end of the procedure. The patient had no complications and was discharged with a modified Rankin Scale score of 0 (Fig. 2A-2C).

\section{Case 2}

A 50-year-old man presenting with a sudden onset of headache was admitted to our hospital. CT revealed a Fisher group 3 subarachnoid hemorrhage, and digital subtraction angiography revealed a left MCA aneurysm. On admission, the patient was classified as Grade I according to World Federation of Neurosurgical Societies (WFNS) and Hunt and Kosnik Grading system.
The size of the aneurysm was $8.1 \mathrm{~mm}$ with an irregular shape and a bleb. Endovascular treatment was selected. We inserted Transform $3.0 \mathrm{~mm} \times 5 \mathrm{~mm}$ from the inferior trunk of M2 to the neck of the aneurysm. Then, we inserted an SL-10 at an angle of $45^{\circ}$ into the aneurysm and performed coil embolization by balloon-assisted technique. We used Axium Prime 3D $4.0 \mathrm{~mm} \times 12 \mathrm{~cm}$ as first coil and then used three Axium Prime 3D extrasoft coils $(3.0 \mathrm{~mm} \times 6 \mathrm{~cm})$, four Axium Prime 3D extrasoft coils $(2.0 \mathrm{~mm} \times 3 \mathrm{~cm})$, an Axium Prime 3D extrasoft coil $(1.0 \mathrm{~mm} \times 3 \mathrm{~cm})$, and an Axium Prime 3D extrasoft coil $(1.0 \mathrm{~mm} \times 2 \mathrm{~cm})$. The aneurysm was completely obliterated. The patient had no complications and was discharged with a modified Rankin Scale score of 0 . Retreatment of the aneurysm was not required during follow-up angiography at 6 months postcoil embolization (Fig. 3A-3D). 

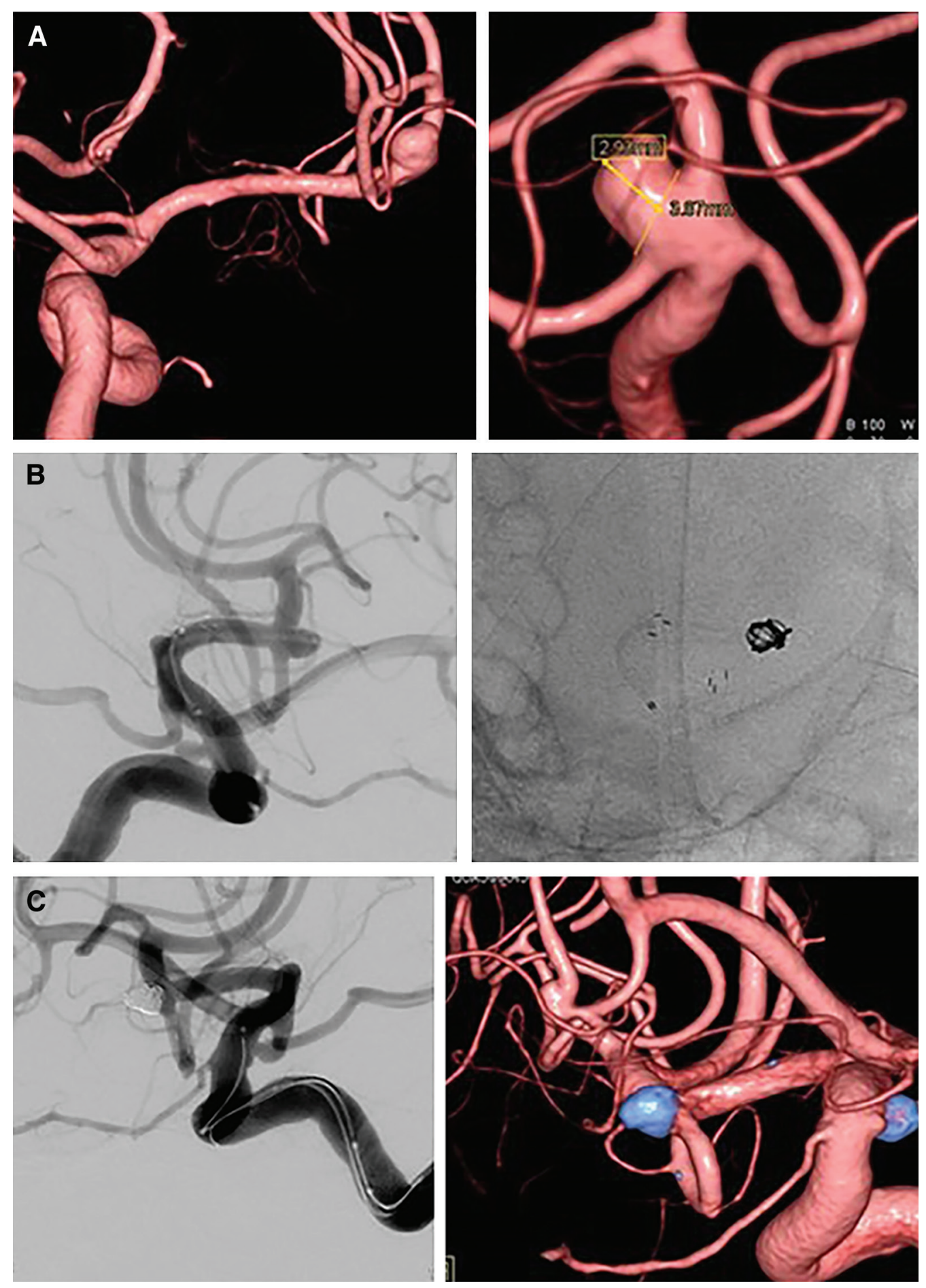

Fig. 2 (A) Case of a 75-year-old woman with unruptured MCA aneurysm. The size of the aneurysm was $2.9 \mathrm{~mm}$ and the neck of the aneurysm was $3.9 \mathrm{~mm}$. (B) SL-10 $45^{\circ}$ was inserted into the aneurysm and the Neuroform Atlas $3.0 \mathrm{~mm} \times$ $21 \mathrm{~mm}$ was deployed to cover the neck. (C) The aneurysm was completely occluded. MCA: middle cerebral artery

\section{Discussion}

In this study, we studied safety and efficacy of endovascular treatment for MCA aneurysms.

The outcomes of clipping of MCA aneurysms were reported to be excellent, with morbidity and mortality rates of $2 \%-6 \%$ and $0 \%-2 \%$, respectively. ${ }^{3-5)}$ Thus, the clipping remains the mainstay for the treatment for MCA aneurysms in many institutions. However, aging and increasing size of aneurysms are reported to contribute to morbidity and mortality. ${ }^{4)}$ Therefore, endovascular treatment might be considered for the treatment of MCA aneurysms, especially in the elderly. Regli et al. compared the outcomes and the technical success rates between endovascular treatment and clipping. 
A

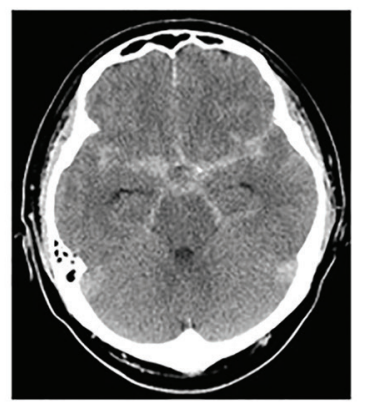

C

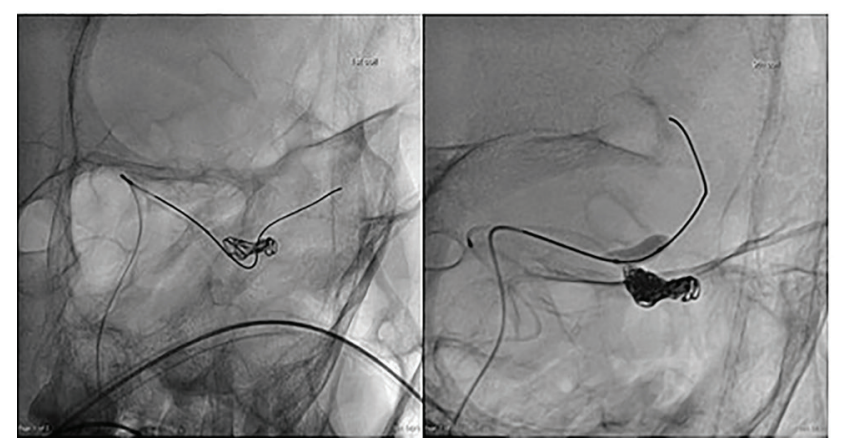

Fig. 3 (A) Case of a 50-year-old man with a ruptured MCA aneurysm. CT at admission revealed subarachnoid hemorrhage (Fisher group 3). (B) Digital subtraction angiography showed that the size of the aneurysm was $8.1 \mathrm{~mm}$ and the aneurysm was irregularly shaped with a bleb. (C) SL-10 $45^{\circ}$ was inserted into the aneurysm and the

They concluded that clipping appears to be the most efficient treatment option for unruptured MCA aneurysms. However, they only performed a simple technique using GDC coil. ${ }^{2)}$ Recently, Mortimer et al. ${ }^{1)}$ reported that the outcomes of endovascular treatment for ruptured MCA aneurysms were comparable to those of clipping. Thus, recent advancement of endovascular techniques might contribute to safety and efficacy of endovascular treatment for MCA aneurysms.

The technical success rate in our study was $100 \%$ and the rates of complete occlusion, neck remnants, and aneurysm remnants were $84.6 \%, 15.4 \%$, and $0 \%$, respectively. These rates were excellent compared to previous studies. Mortimer et al. ${ }^{1}$ reported a technical success rate of $94.4 \%$, in which $71.7 \%$ aneurysms were completely occluded and $19.7 \%$ exhibited neck remnants. In addition, a systematic review for endovascular treatment for more than 1000 MCA aneurysms also reported that $82.4 \%$ of MCA aneurysms were completely occluded or nearly completely occluded. ${ }^{10)}$ At our institution, we performed endovascular treatment for selected MCA aneurysms. We thought endovascular treatment of Type II aneurysms was safe because catheterization and coil insertion were easy and the microcatheter was stable during the procedure. As a result, the
B

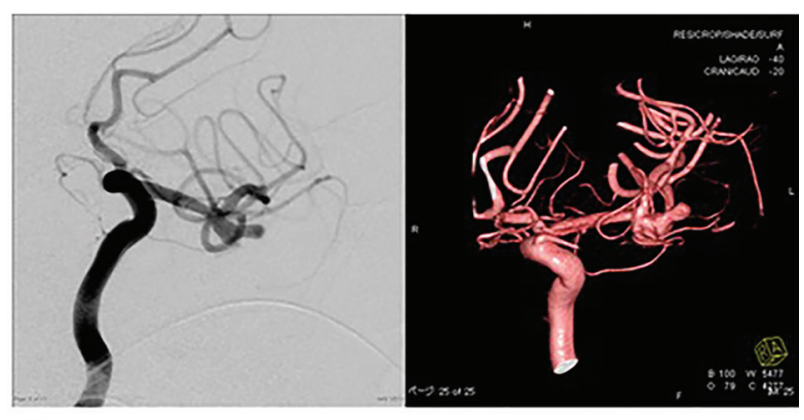

D

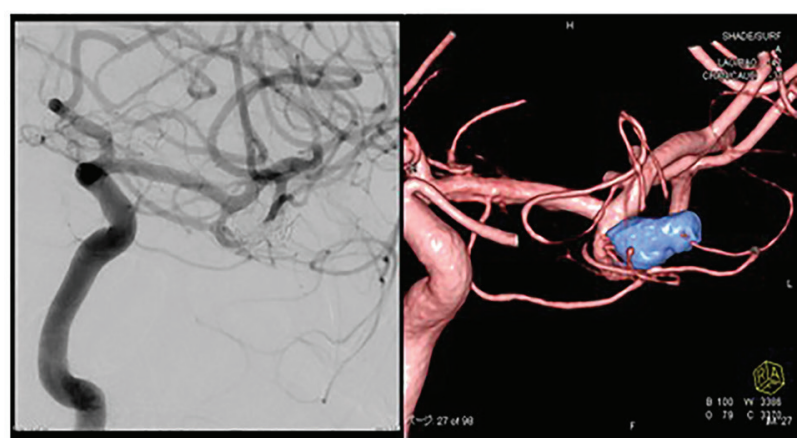

coil embolization with balloon assist technique was performed. A transform $3 \mathrm{~mm} \times 5 \mathrm{~mm}$ were used to cover the neck. (D) Follow-up digital subtraction angiography at 6 months after the initial coil embolization. The aneurysm was still completely occluded. MCA: middle cerebral artery

proportion of Type II aneurysms was the highest among the all three types of MCA aneurysms treated with endovascular treatment. In addition, Type II aneurysms with round shape were more likely treated with endovascular treatment than irregular shaped Type II aneurysms.

We thought careful selection of endovascular treatment for MCA aneurysms would contribute to the high technical success rate of this study.

Previous studies reported re-treatment rate with a range of $2.4 \%-13.9 \%$ for endovascular treatment for MCA aneurysms. ${ }^{11-14)}$ In our series, the re-treatment rate was $0 \%$; however, only $57.7 \%$ of patients underwent follow-up angiography after 1 year of endovascular procedure, which is lower than previous reports $(60.9 \%-86.8 \%)$. Thus, more complete angiographical follow-up data are necessary to derive a conclusion.

We also compared the morphology of the aneurysms treated with endovascular treatment with those treated by clipping. In most cases, surgical clipping tends to be selected when MCA aneurysms were Type III. We thought these aneurysms were at high risk for endovascular treatment because of difficult catheterization, difficult coil insertion, and unstable microcatheter during the procedure. Thus, in such aneurysms, we chose surgical clipping as a first choice. On the other hand, 
when the aneurysm was Type II, both surgical clipping and endovascular coiling may be equally chosen.

In addition, five patients with coil embolization had a Type I aneurysm in our series and all of them were treated with endovascular treatment. These aneurysms need to be discussed differently from the bifurcation type MCA aneurysms. Previous reports suggested that these aneurysms were technically challenging to treat by clipping, as these aneurysms were usually located at the origin of the lenticulostriate arteries and these arteries had to be dissected from the aneurysms. ${ }^{15,16)} \mathrm{In}$ contrast, favorable outcomes for endovascular treatment of proximal MCA (M1) aneurysms were also reported. ${ }^{17-19)}$ Thus, we suggest that endovascular treatment may be the first choice for the treatment of proximal MCA (M1) aneurysms.

\section{Conclusion}

The results from the present study identify coil embolization combined with assist techniques, such as the balloon-assisted and the stent-assisted techniques, are safe as a treatment for MCA aneurysm with acceptable complication rates. In addition, M1 trunk aneurysms and MCA bifurcation aneurysms with a round shape located along the same axis of M1 trunk may be a good indication for endovascular treatment.

\section{Disclosure Statement}

Author Toshiyuki Fujinaka received lecture fees from Stryker and Medtronic. The other authors have no conflict of interest.

\section{References}

1) Mortimer AM, Bradley MD, Mews P, et al: Endovascular treatment of 300 consecutive middle cerebral artery aneurysms: clinical and radiologic outcomes. AJNR Am J Neuroradiol 2014; 35: 706-714.

2) Regli L, Uske A, de Tribolet N: Endovascular coil placement compared with surgical clipping for the treatment of unruptured middle cerebral artery aneurysms: a consecutive series. J Neurosurg 1999; 90: 1025-1030.

3) Choi SW, Ahn JS, Park JC, et al: Surgical treatment of unruptured intracranial middle cerebral artery aneurysms: angiographic and clinical outcomes in 143 aneurysms. $J$ Cerebrovasc Endovasc Neurosurg 2012; 14: 289-294.

4) Morgan MK, Mahattanakul W, Davidson A, et al: Outcome for middle cerebral artery aneurysm surgery. Neurosurgery 2010; 67: 755-761; discussion 761.

5) Rodríguez-Hernández A, Sughrue ME, Akhavan S, et al: Current management of middle cerebral artery aneurysms: surgical results with a "clip first" policy. Neurosurgery 2013; 72: 415-427.

6) van Dijk JM, Groen RJ, Ter Laan M, et al: Surgical clipping as the preferred treatment for aneurysms of the middle cerebral artery. Acta Neurochir (Wien) 2011; 153: 2111-2117.

7) Gory B, Rouchaud A, Saleme S, et al: Endovascular treatment of middle cerebral artery aneurysms for 120 nonselected patients: a prospective cohort study. AJNR Am J Neuroradiol 2014; 35: 715-720.

8) Kadkhodayan Y, Delgado Almandoz JE, Fease JL, et al: Endovascular treatment of 346 middle cerebral artery aneurysms: results of a 16-year single-center experience. Neurosurgery 2015; 76: 54-60; discussion 60-61.

9) Roy D, Milot G, Raymond J: Endovascular treatment of unruptured aneurysms. Stroke 2001; 32: 1998-2004.

10) Brinjikji W, Lanzino G, Cloft HJ, et al: Endovascular treatment of middle cerebral artery aneurysms: a systematic review and single-center series. Neurosurgery 2011; 68: 397-402; discussion 402.

11) Quadros RS, Gallas S, Noudel R, et al: Endovascular treatment of middle cerebral artery aneurysms as first option: a single center experience of 92 aneurysms. AJNR Am J Neuroradiol 2007; 28: 1567-1572.

12) Suzuki S, Tateshima S, Jahan R, et al: Endovascular treatment of middle cerebral artery aneurysms with detachable coils: angiographic and clinical outcomes in 115 consecutive patients. Neurosurgery 2009; 64: 876-888; discussion 888-889.

13) Bracard S, Abdel-Kerim A, Thuillier L, et al: Endovascular coil occlusion of 152 middle cerebral artery aneurysms: initial and midterm angiographic and clinical results. J Neurosurg 2010; 112: 703-708.

14) Oishi H, Yoshida K, Shimizu T, et al: Endovascular treatment with bare platinum coils for middle cerebral artery aneurysms. Neurol Med Chir (Tokyo) 2009; 49: 287-293.

15) Ha SK, Lim DJ, Kang SH, et al: Analysis of multiple factors affecting surgical outcomes of proximal middle cerebral artery aneurysms. Clin Neurol Neurosurg 2011; 113: 362-367.

16) Hosoda K, Fujita $S$, Kawaguchi $T$, et al: Saccular aneurysms of the proximal (M1) segment of the middle cerebral artery. Neurosurgery 1995; 36: 441-446.

17) Zhou Y, Yang PF, Fang YB, et al: Endovascular treatment for saccular aneurysms of the proximal (M1) segment of the middle cerebral artery. Acta Neurochir (Wien) 2012; 154: 1835-1843.

18) Lubicz B, Pezzullo M, Brisbois D, et al: Endovascular treatment of proximal superior middle cerebral artery aneurysms. Neuroradiology 2012; 54: 1267-1273.

19) Cho YD, Lee WJ, Kim KM, et al: Endovascular coil embolization of middle cerebral artery aneurysms of the proximal (M1) segment. Neuroradiology 2013; 55: 1097-1102. 